\title{
Prevalência e prognóstico de desnutrição determinados pelo critério GLIM
}

\author{
Prevalence and nutrition prognostics determined by GLIM criteria
}

\section{DOI: $10.37111 /$ braspenj.2020351009}

Bianca Cristina Laty'

Gabriela Datsch Bennemann²

Mariana Abe Vicente Cavagnari ${ }^{3}$

Angélica Rocha de Freitas Melhem ${ }^{4}$

Caryna Eurich Mazur ${ }^{5}$

Dalton Luiz Schiessel ${ }^{6}$

\section{Unitermos:}

Dietoterapia. Avaliação nutricional. Estilo de vida. Desnutrição.

\section{Keywords:}

Diet therapy. Nutrition assessment. Life style. Malnutrition.

\section{Endereço para correspondência:}

Dalton Luiz Schiessel

Universidade Estadual do Centro-Oeste Campus

CEDETEG - Departamento de Nutrição

Alameda Élio Antonio Dalla Vecchia, 838 - Vila Carli

- Guarapuava, PR, Brasil - CEP 85040-167

E-mail: daltonls68@gmail.com

\section{Submissão}

20 de setembro de 2019

Aceito para publicação

25 de março de 2020

\begin{abstract}
Resumo
Introdução: A desnutrição está presente entre $25 \%$ a $70 \%$ dos pacientes hospitalizados, podendo predizer aumento no risco de complicações, redução na resposta ao tratamento, menor qualidade de vida, aumento dos custos e tempo de hospitalização e aumento da mortalidade. $\mathrm{O}$ objetivo deste estudo foi aplicar o Global Leadership Initiative on Malnutrition (GLIM) em pacientes hospitalizados e verificar a associação com idade, sexo, diferentes patologias e consumo alimentar. Método: Estudo longitudinal e de caráter retrospectivo. Foram coletados dados sociodemográficos, antropométricos, clínicos e alimentares dos prontuários de atendimento nutricional de pacientes adultos, avaliados entre os anos de 2015 a 2018, em dois hospitais com serviços e clínicas especializadas que atendem ao Sistema Único de Saúde (SUS). Foi utilizado o modelo de regressão logística multinomial (RLM) univariada e multivariada para determinar o risco relativo (odds ratio), ajustados os desfechos para as variáveis: idade, sexo, patologias e consumo alimentar e comparadas com a classificação de GLIM. Resultados: Os pacientes apresentaram redução de peso significativa (peso usual para peso atual) e \% perda de peso de $-7,6 \%(95 \%$ IC $-8,4--6,8)$. Idade, patologias, presença de inflamação e redução do consumo alimentar são contribuintes para a perda de peso e a presença de desnutrição. As doenças predominantes são altamente catabólicas e possuem resposta elevada para a presença de inflamação sistêmica, especificamente os pacientes com câncer, que apresentaram na análise multivariada chances de 2,21 vezes de estarem com desnutrição moderada e 2,72 vezes com desnutrição grave. Conclusão: A prevalência de desnutrição foi $46,8 \%$ do total de pacientes com risco relativo significativo com relação à idade, principalmente com diagnóstico de câncer e quando relatado o consumo alimentar reduzido/inadequado.
\end{abstract}

\section{ABSTRACT}

Introduction: Malnutrition is present in $25 \%$ to $70 \%$ of hospitalized patients, being caused by factors such as may predict an increased risk of complications, reduced response to treatment, lower quality of life, increased costs and length of hospitalization and increased mortality. Thus, the objective was to apply Global Leadership Initiative on Malnutrition (GLIM) to hospitalized patients and the association with age, gender, different pathologies and food intake. Methods: Longitudinal and retrospective study. Sociodemographic, anthropometric, clinical and dietary data were collected from adult patient nutritional records, evaluated between 2015 and 2018, in two hospitals with specialized services and clinics that serve the Unified Health System (SUS). The univariate and multivariate multinomial logistic regression model (RLM) was used to determine the relative risk (odds ratio), adjusted for the following variables: age, sex, pathologies and food intake and compared with the GLIM classification. Results: Patients had significant weight reduction (usual weight to current weight) and \% weight loss of $-7.6 \%$ (95\% $\mathrm{Cl}-8.4--6.8)$. Age, pathologies, presence of inflammation, and reduced food intake all contribute to weight loss and malnutrition. The predominant diseases are highly catabolic and have a high response to the presence of systemic inflammation, specifically cancer patients who presented in the multivariate analysis chances of 2.21 times of being with moderate malnutrition and 2.72 times severe malnutrition. Conclusion: The prevalence of malnutrition was $46.8 \%$ of the total patients with significant relative risk regarding age, especially with cancer diagnosis and when reported reduced/inadequate food intake.

1. Graduada em Nutrição, Universidade Estadual do Centro Oeste, Guarapuava, PR, Brasil.

2. Nutricionista, Doutora em Ciências Agrárias, Professora do Departamento de Nutrição da Universidade Estadual do Centro Oeste (UNICENTRO), Guarapuava, PR, Brasil.

3. Nutricionista, Doutora em Ciências pelo Programa de Pós-Graduação em Gastroenterologia pela Universidade Federal de São Paulo, Professora do Departamento de Nutrição da Universidade Estadual do Centro Oeste (UNICENTRO), Guarapuava, PR, Brasil.

4. Nutricionista, Doutora em Ciências pelo Programa de Pós-Graduação em Gastroenterologia pela Universidade Federal de São Paulo, Professora do Departamento de Nutrição da Universidade Estadual do Centro Oeste (UNICENTRO), Guarapuava, PR, Brasil.

5. Nutricionista, Doutora em Medicina Interna e Ciências da Saúde pela Universidade Federal do Paraná, Professora do Departamento de Nutrição da Universidade Estadual do Centro Oeste (UNICENTRO), Guarapuava, PR, Brasil.

6. Nutricionista, Doutor em Biologia Celular e Molecular pela Universidade Federal do Paraná, Pós-Doutorado pela University of Alberta - Cross Cancer Institute, Edmonton - Canadá, Professor do Departamento de Nutrição da Universidade Estadual do Centro Oeste (UNICENTRO), Guarapuava, PR, Brasil. 


\section{INTRODUÇ̃̃O}

A promoção à saúde compreende um conjunto de estratégias que objetivam proporcionar aos indivíduos e coletividades a realização de práticas alimentares apropriadas, visando à prevenção do risco de desenvolver doenças não transmissíveis'. Quando na presença de enfermidades é possível a identificação de pacientes hospitalizados com risco e/ou recuperação do estado nutricional ${ }^{2}$.

No âmbito hospitalar, a desnutrição está presente entre $25 \%$ a $70 \%$ dos pacientes hospitalizados, sendo ocasionada por fatores como aumento das necessidades nutricionais, diminuição da capacidade de digestão, absorção e metabolismo dos nutrientes, períodos prolongados de jejum, restrições alimentares, inapetência, presença de inflamação, entre outros ${ }^{3}$. Isto pode predizer aumento no risco de complicações, redução na resposta ao tratamento, menor qualidade de vida, aumento dos custos e tempo de hospitalização e aumento mortalidade ${ }^{3,4}$.

Identificar pacientes desnutridos ou com risco nutricional permite compreender um processo de ir ao encontro das diferentes necessidades nutricionais, que inclui a avaliação do estado nutricional, a identificação de riscos e carências, o planejamento de objetivos e intervenções do cuidado nutricional que preencham todas as necessidades, para um tratamento pertinente e fidedigno ${ }^{5}$. Relacionar os métodos de avaliação do perfil nutricional com as patologias dos pacientes auxilia o nutricionista a reverter o processo patológico, que pode levá-lo à desnutrição e à reinternação ${ }^{6}$.Para reforçar esse cuidado e padronizar o diagnóstico de desnutrição, as sociedades de Nutrição Clínica, American Society for Parenteral and Enteral Nutrition (ASPEN), (European Society for Clinical Nutrition and Metabolism (ESPEN), Federación Latino-Americana de Terapia Nutricional, Nutrición Clinica y Metabolismo (FELANPE) e Parenteral and Enteral Nutrition Society of Asia (PENSA), constituíram a Iniciativa de Liderança Global em Desnutrição (Global Leadership Initiative on Malnutrition - GLIM), caracterizada pela aplicação de critérios recentemente desenvolvidos para o diagnóstico de desnutrição em adultos em ambientes clínicos, compreendendo três critérios fenotípicos para graduar a gravidade, desde moderada a importante. Essa ferramenta permite complementar a triagem nutricional, seguida da avaliação nutricional completa do paciente, assumindo fundamental importância de ampliação e auxílio dentro do processo do cuidado nutricional e intervenções ${ }^{7}$.

Nesse contexto, o objetivo do estudo foi avaliar o estado nutricional de pacientes no âmbito hospitalar, traçando o perfil nutricional e clínico (antropométrico, alimentar e patologias associadas), avaliar a perda de peso, triagem nutricional, hábitos de vida e correlacionar com as patologias, aplicando os critérios GLIM.

\section{MÉTODO}

Caracteriza-se como um estudo de coorte, de ação longitudinal e de caráter retrospectivo, com a inclusão de pacientes adultos de ambos os gêneros, avaliados entre os anos de 2015 a 2018, em dois hospitais com serviços e clínicas especializadas que atendem ao Sistema Único de Saúde (SUS), na região de Guarapuava-PR. Os critérios de exclusão foram os prontuários com preenchimento incorreto ou com ausência de dados. A pesquisa foi aprovada pelo Comitê de Ética em Pesquisa com Seres Humanos da Universidade Estadual do Centro-Oeste (UNICENTRO), sob o parecer número 1593833/2016.

Foram coletados os dados sociodemográficos (sexo, idade, etilismo, tabagismo e escolaridade), antropométricos (histórico do peso com 6 meses de antecedência, peso atual e estatura), clínicos (diagnóstico clínico e patologias associadas), a limentar (dieta prescrita, aceitação e alteração do consumo alimentar) e dados da triagem Nutritional Risk Screening (NRS 2002)', dos prontuários de atendimento nutricional de pacientes com diversas doenças não transmissíveis (DNT).

O estado nutricional de acordo com o índice de massa corporal (IMC) foi classificado de acordo com a Organização Mundial da Saúde e o percentual de perda de peso foi calculado pela fórmula (peso atual/peso usual) x 100 .

Após as coletas dos dados antropométricos e alimentar, foram aplicados os critérios de GLIM para a classificação e graduação da gravidade de desnutrição, os quais compreendem três critérios fenotípicos: perda de peso (> $5 \%$ nos últimos 6 meses, ou> 10\% além dos 6 meses), baixo IMC ( $<20$ se $<70$ anos, ou $<22$ se $>70$ anos / Ásia: $<18,5$ se $<70$ anos, ou $<20$ se $>70$ anos), massa muscular reduzida (quando reduzida por técnicas validadas de medição da composição corporal) e dois critérios etiológicos: redução da ingestão alimentar ( $\leq 50 \%$ da redução alimentar $>1$ semana, ou qualquer redução por $>2$ semanas, ou qualquer condição que cause impacto adverso na ingestão ou absorção de alimentos) e presença de inflamação (relacionada à doença/ lesão aguda ou doença crônica). Considerando a descrição desses critérios, para o paciente ser diagnosticado com desnutrição necessita conter a presença de um critério fenotípico e um etiológico?.

Para graduar a gravidade da desnutrição, GLIM considera apenas os critérios fenotípicos, sendo classificados com desnutrição moderada, aqueles que possuem perda de peso de $5 \%$ a $10 \%$, nos últimos 6 meses, ou $10 \%$ a $20 \%$, após 6 meses, IMC $<20$ se $<70$ anos, $<22$ se $\geq 70$ anos e massa muscular reduzida em déficit leve a moderado por métodos validados. Classifica-se como desnutrição importante aqueles que possuem perda de peso $>10 \%$, nos últimos 6 meses, ou $>20 \%$, além dos 6 meses, IMC $<18,5$ se $<70$ anos, $<20$ se $\geq 70$ anos e massa muscular reduzida em déficit grave ${ }^{7,9}$. 
Os dados foram analisados descritivamente, com a utilização de médias, desvio padrão e frequências relativas e absolutas. $O$ principal desfecho é a relação com a perda de peso e a classificação GLIM, para o qual foi realizado teste de normalidade e homogeneidade dos dados (ShapiroWilk), sendo então aplicado o teste estatístico apropriado. Para comparação das variáveis numéricas foi realizado teste t de Student para amostras independentes ou teste de Mann-Whitney. A comparação das variáveis qualitativas foi realizada por meio do teste qui-quadrado de Pearson. Foi utilizado o modelo de regressão logística multinomial (RLM) univariada e multivariada para determinar o risco relativo (odds ratio), ajustados os desfechos para as variáveis: idade, sexo, patologias e consumo alimentar e comparadas com a classificação do GLIM Nutrido. O intervalo de confiança de 95\% (IC) foi adotado para o risco relativo estimado calculado. Todas as análises foram feitas com o auxílio do software IBM-SPSS ${ }^{\circledR}$ Statistics 22 .

\section{RESULTADOS}

Foram analisados 1.389 prontuários, sendo o sexo feminino predominante e média de idade de 60,5 anos (95\% IC 59,6 - 61,4). Os pacientes apresentaram redução significante do peso corporal e \% perda de peso de $-7,6 \%$ (95\% IC -8,4 - -6,8). Apesar dos homens apresentarem maior percentual de perda de peso $(8,6 \%)$, não foi observada diferença significativa entre as mulheres $(6,6 \%)$. Posteriormente, houve redução no IMC, o que permite observar significativa alteração do estado nutricional, com aumento do número de pacientes classificados com baixo peso em aproximadamente 3 vezes (Tabela 1).
Em relação aos dados sociodemográficos, destacou-se as características: $13,3 \%(n=180)$ etilista, $26,3 \%(n=356)$ tabagista, $43 \%(n=346)$ possuem ensino fundamental incompleto e 19,3\% $(n=155)$ médio completo. Em relação aos antecedentes patológicos familiares, 17,4\% ( $n=208)$ possuem câncer, $31,8 \%(n=385)$ diabetes mellitus, 42,4\% $(n=511)$ hipertensão arterial. Após o internamento, 78, 1\% dos pacientes sofreram alterações na própria alimentação, passando $46 \%$ ( $n=639$ ) para uma dieta branda, a qual se caracteriza por ser uma dieta com baixo teor de celuloses (fibras) e tecidos conjuntivos modificados por meio da cocção.

A clínica de internação predominante foi a médica (79\%) e todos os pacientes estavam em tratamento, com incidência de $65 \%$ para doenças cardiovasculares e associadas, doenças do trato gastrointestinal e anexos, câncer e doença pulmonar, sendo a perda de peso maior para esses pacientes, como também aqueles em tratamento para doenças neurológicas (Tabela 2).

Na Tabela 3, os pacientes foram estratificados aplicando o critério GLIM e 651 (46,9\%) foram classificados como desnutridos e não houve diferenças na prevalência de desnutrição moderada ou grave entre os sexos. $O$ peso atual foi significativamente diferente entre os grupos, sendo menor para o grupo desnutrido grave, assim como o IMC atual, o \% de perda de peso e o consumo alimentar alterado, alta incidência foi observada nos pacientes desnutridos moderados e graves, pois estes três últimos são critérios da classificação do GLIM. Quando comparado com as patologias, o quadro de desnutrição moderada e grave teve grande predominância para o câncer, doença pulmonar e outras patologias.

Tabela 1 - Características demográficas e antropométricas de pacientes internados nos hospitais de Guarapuava-PR.

\begin{tabular}{|c|c|c|c|c|}
\hline Características demográficas / clínicas & Todos pacientes & Masculino & Feminino & $p$ \\
\hline Sexo - n (\%) & $1389(100 \%)$ & $673(48,5 \%)$ & $716(51,5 \%)$ & - \\
\hline Idade (anos) & $60,5 \pm 17,1$ & $60,4 \pm 16,9$ & $60,6 \pm 17,4^{*}$ & 0,816 \\
\hline Estatura (m) & $1,63 \pm 0,1$ & $1,69 \pm 0,08$ & $1,58 \pm 0,08^{*}$ & $<0,001$ \\
\hline $\begin{array}{l}\text { Peso usual }(\mathrm{kg}) \\
\text { Peso atual }(\mathrm{kg})\end{array}$ & $\begin{array}{c}69,9 \pm 17,2 \\
64,7 \pm 18,2 \#\end{array}$ & $\begin{array}{c}74,4 \pm 17,4 \\
68,6 \pm 18,1 \#\end{array}$ & $\begin{array}{l}65,7 \pm 15,9^{*} \\
61,0 \pm 17,6 \#^{*}\end{array}$ & $\begin{array}{l}<0,001 \\
<0,001\end{array}$ \\
\hline$\%$ de perda de peso (\%) & $-7,6 \% \pm 12,2$ & $-8,6 \pm 12,2$ & $-6,6 \pm 12,2^{\star}$ & 0,091 \\
\hline \multicolumn{5}{|l|}{ Classificação IMC usual - n (\%) } \\
\hline Baixo peso & $80(5,8 \%)$ & $33(4,9 \%)$ & $47(6,6 \%)$ & \\
\hline Eutrófico & $440(31,7 \%)$ & $233(34,6 \%)$ & $207(28,9 \%)$ & \\
\hline Sobrepeso & $342(24,6 \%)$ & $157(23,3 \%)$ & $185(25,8 \%)$ & \\
\hline Obesidade & $227(16,3 \%)$ & $106(15,8 \%)$ & $121(16,9 \%)$ & \\
\hline Obesidade & $197(14,2 \%)$ & $79(11,7 \%)$ & $118(16,5 \%)$ & \\
\hline
\end{tabular}


Laty BC et al.

Tabela 2 - Características clínicas, \% da perda de peso e distribuição de pacientes por sexo de acordo com as patologias

\begin{tabular}{|c|c|c|c|c|}
\hline Patologias & $\begin{array}{c}\% \text { perda de peso } \\
(\%)\end{array}$ & $\begin{array}{c}\text { Todos pacientes } \\
\mathrm{n}(\%) \\
\end{array}$ & $\begin{array}{c}\text { Masculino } \\
\mathrm{n}(\%) \\
\end{array}$ & $\begin{array}{c}\text { Feminino } \\
\mathrm{n}(\%)\end{array}$ \\
\hline Sepse e trauma & $-3,4 \pm 8,7$ & $22(1,6 \%)$ & $11(1,6 \%)$ & $11(1,5 \%)$ \\
\hline Anemia & $-6,4 \pm 13,0$ & $35(2,5 \%)$ & $8(1,2 \%)$ & $27(3,8 \%)$ \\
\hline Doença renal / excretor & $-4,9 \pm 12,3$ & $38(2,7 \%)$ & $15(2,2 \%)$ & $23(3,25)$ \\
\hline $\begin{array}{l}\text { Insuficiência renal } \\
\text { Doenças neurológicas }\end{array}$ & $\begin{array}{l}-6,9 \pm 10,3 \\
-9,5 \pm 11,9\end{array}$ & $\begin{array}{l}39(2,8 \%) \\
68(4,9 \%)\end{array}$ & $\begin{array}{l}20(3,0 \%) \\
36(5,3 \%)\end{array}$ & $\begin{array}{l}19(2,7 \%) \\
32(4,5 \%)\end{array}$ \\
\hline Doença cardiovascular/HAS/DM & $-4,2 \pm 10,3$ & $174(12,5 \%)$ & $92(13,7 \%)$ & $82(11,5 \%)$ \\
\hline Doença do TGI / anexos & $-9,7 \pm 11,7$ & $201(14,5 \%)$ & $98(14,6 \%)$ & $103(14,4 \%)$ \\
\hline Câncer & $-10,6 \pm 13,6$ & $257(18,5 \%)$ & $146(21,7 \%)$ & $111(15,5 \%)$ \\
\hline Doença pulmonar & $-7,3 \pm 12,8$ & $269(19,4 \%)$ & $113(16,8 \%)$ & $156(21,8 \%)$ \\
\hline Outras & $-5,6 \pm 10,6$ & $286(20,6 \%)$ & $134(19,9 \%)$ & $152(21,2 \%)$ \\
\hline
\end{tabular}

DM = diabetes mellitus; HAS = hipertensão arterial sistêmica; TGI = trato gastrointestinal.

Tabela 3 - Características demográficas e clínicas de pacientes classificados de acordo com o critério GLIM.

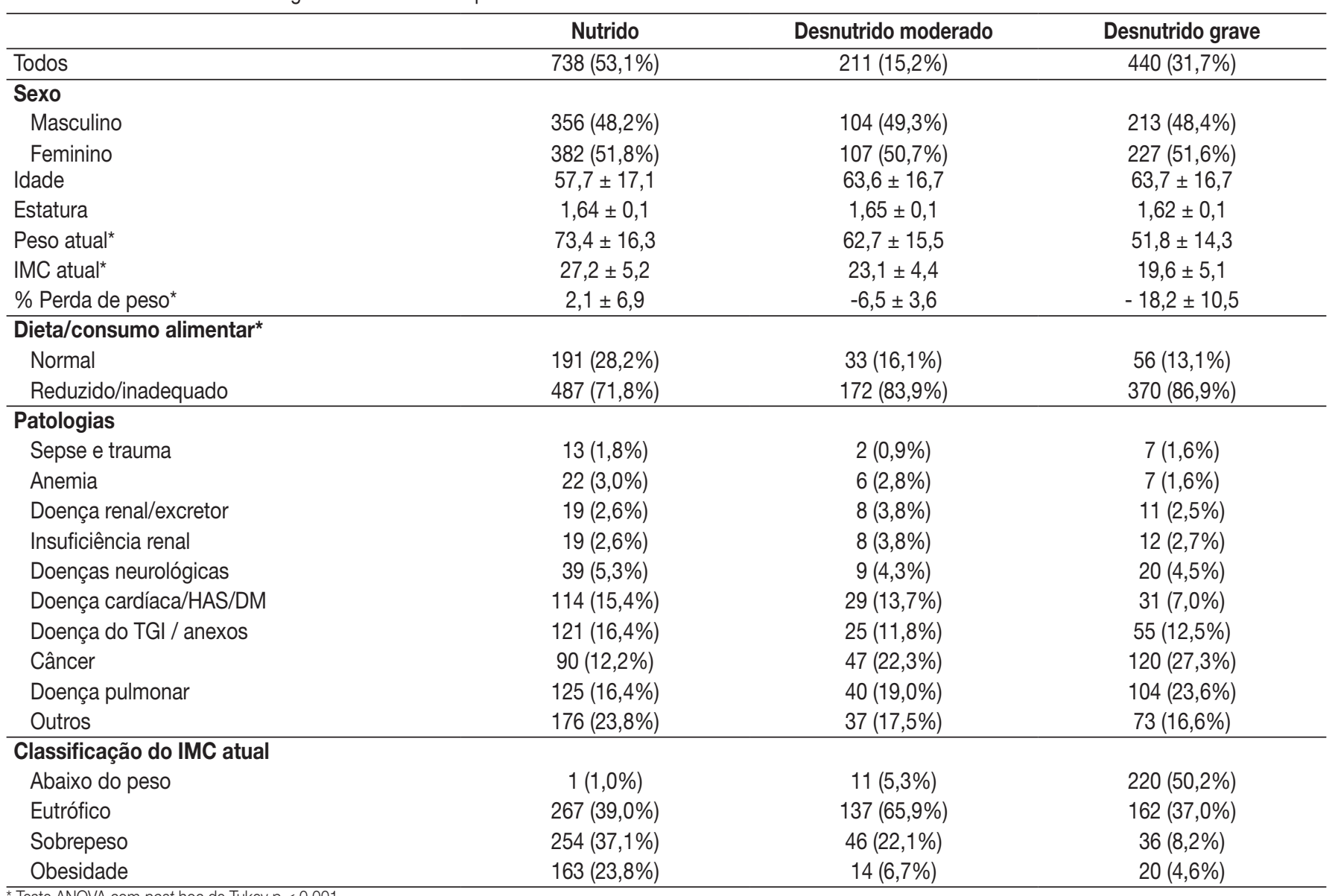

*Teste ANOVA com post hoc de Tukey $p<0,001$.

$\mathrm{DM}$ = diabetes mellitus; IMC = índice de massa corporal; HAS = hipertensão arterial sistêmica; TGl = trato gastrointestinal.

$\mathrm{Na}$ análise univariada, idade, câncer e consumo alimentar reduzido/inadequado foram significantemente associados com desnutrição moderada à grave e doença pulmonar à desnutrição grave comparados com os pacientes classificados como nutridos. No modelo multivariado da RLM, após ajustados para idade, sexo, patologias e consumo alimentar comparados com a classificação do GLIM Nutrido, a variável sexo apresentou RR 1,02 (95\% IC 1,01 - 1,03), doença renal - excretor, o RR foi 2,86
(95\% IC 1,10 - 7,41), câncer o RR 2,21 (95\% IC 1,31 3,73 ) e consumo alimentar reduzido/inadequado o RR foi 1,99 (95\% IC 1,31 - 3,03) para a desnutrição moderada. Já para a classificação do GLIM desnutrido grave para a idade, o RR foi 1,02 (95\% IC 1,01 - 1,03), nas doenças cardiovasculares associadas (DM e HAS), O RR foi 0,50 (95\% IC 0,30 - 0,83). O câncer apresentou RR 2,72 (95\% IC 1,81-4,10) e consumo alimentar reduzido/inadequado o RR foi 2,48 (95\% IC 1,76-3,49) (Tabela 4). 
Tabela 4 - Análise da regressão logística multinomial (RLM) correlacionada com a classificação do GLIM.

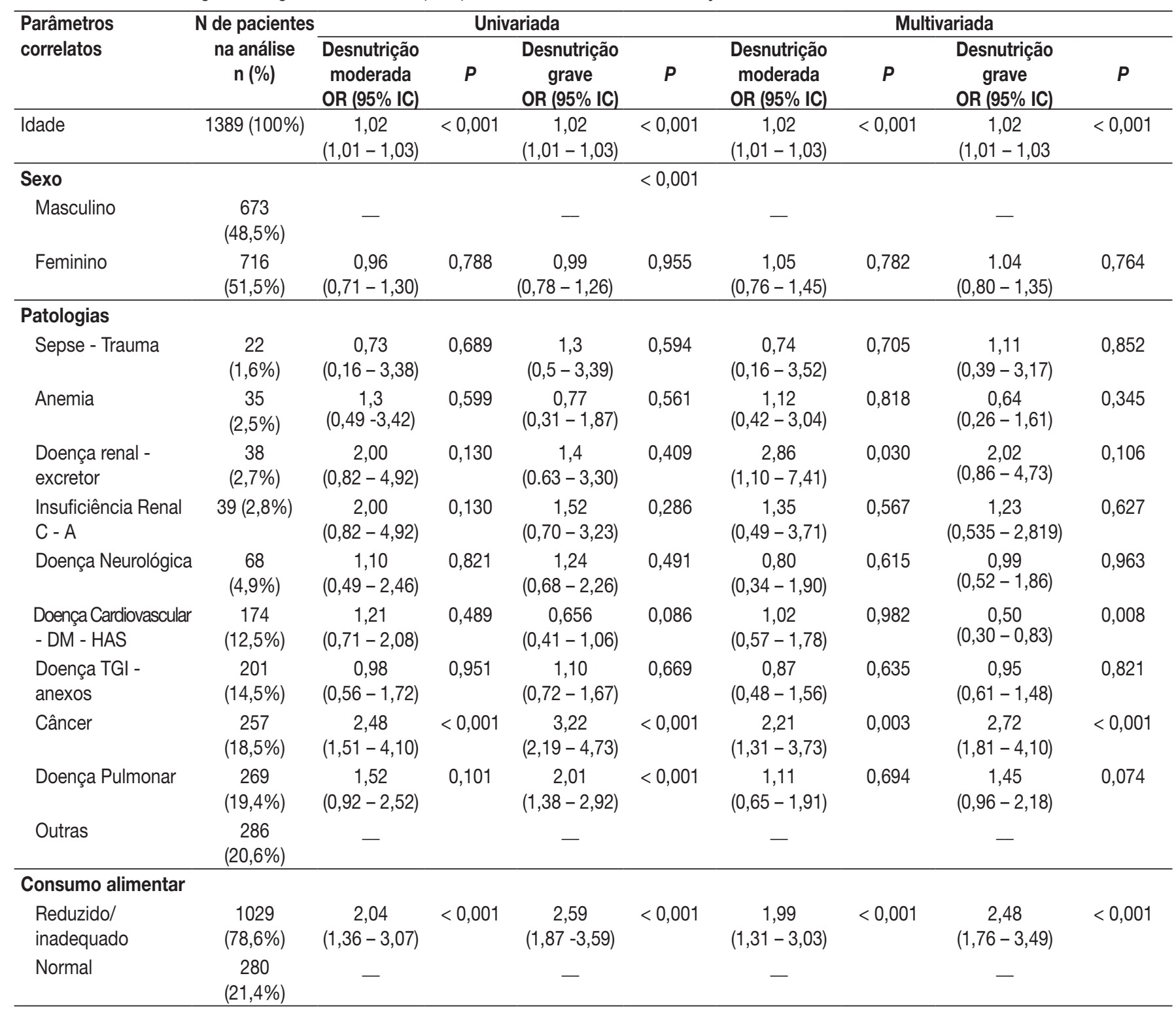

Interceptação em todas as análises $p<0,001$. A categoria de referência: nutrido.

$\mathrm{DM}$ = diabetes mellitus; HAS = hipertensão arterial sistêmica; TGI = trato gastrointestinal.

\section{DISCUSSÃO}

O GLIM possui vários domínios, garantindo maior especificidade pela inclusão dos critérios fenotípicos (perda de peso, IMC e redução da massa muscular) e etiológicos (redução da ingestão alimentar e presença de inflamação associada à doença) para um diagnóstico completo da desnutrição. Como base para a classificação da gravidade em moderada ou grave, utilizam-se pontos de corte específicos dos critérios fenotípicos ${ }^{7}$.

A aplicação dessa ferramenta tem o objetivo de promover o uso global pelos profissionais da área da saúde ${ }^{7}$. Esse método é aplicado individualmente, baseado na disponibilidade dos recursos para aplicação de todos os critérios, recomendando-se a avaliação frequente para a manutenção do estado nutricional e identificação de melhorias?. Ainda são poucos os estudos que validam a utilização dos critérios GLIM, usando métodos simples e ampliando o diagnóstico de desnutrição e o avanço para um tratamento procedente ${ }^{10}$.

A desnutrição relacionada às doenças é uma condição prevalente entre pacientes em locais que englobam o cuidado à saúde. Sua causalidade multifatorial pode ser explicada por um comprometimento na ingestão ou assimilação de nutrientes, inflamação aguda ou crônica, alterando o metabolismo com elevação do gasto energético em repouso e aumento do catabolismo muscular e lipídico ${ }^{11}$. A prevalência mundial é reportada em $25 \%$ para mais de $70 \%$, de acordo com diferentes ferramentas de avaliação nutricional ${ }^{12}$. 
Neste estudo, 16,7\% dos pacientes apresentavam-se com baixo peso, de acordo com o IMC atual. Essa ferramenta tende a incluir pacientes com desnutrição na classificação de eutrofia, sobrepeso e obesidade, devido a sua peculiaridade em avaliar a composição corporal somente pelo peso e estatura. Apesar do IMC ser um parâmetro facilmente aplicável e validado, não deve ser utilizado como uma única ferramenta para diagnosticar desnutrição em âmbito hospi$\operatorname{talar}^{2,13}$. Desta forma, recomenda-se a utilização de outros métodos e biomarcadores para complementar o diagnóstico, levando em consideração mais características de pacientes clínicos, como a Avaliação Subjetiva Global (ASG) e/ou Mini Avaliação Nutricional (MAN), exames laboratoriais, como albumina e hemoglobina e/ou GLIM 9,14.

Com a utilização do critério GLIM para o diagnóstico de desnutrição em adultos em ambientes clínicos, a prevalência de desnutrição moderada foi de 15,1\% ( $n=211)$ e desnutrição grave de $31,7 \%(n=440)$, considerando-se um valor alto quando comparado ao IMC atual de baixo peso. Avaliados pelo GLIM em uma coorte de 6110 pacientes cirúrgicos do trato gastrintestinal, 19,7\% eram desnutridos moderados e 15,6\% desnutridos graves ${ }^{15}$.

A média de idade dos pacientes nutridos foi 57,7 anos e 63,6 anos para os pacientes classificados com desnutrição moderada e grave, destacando-se um público de idosos, sendo as chances de perda de peso maiores, pois a cada ano a mais da idade está associada com a chance do paciente ser classificado com desnutrição moderado/ grave $^{16,17}$, onde há modificações fisiológicas provenientes do envelhecimento, diminuindo o desempenho na musculatura e no mecanismo de deglutição, consequentemente, a perda de massa muscular (sarcopenia) é maior, como também a diminuição da ingestão alimentar ${ }^{8}$. A sarcopenia relacionada à idade pode ser fator conexo à perda de peso, sobretudo a perda de massa muscular ${ }^{18}$, a inflamação ${ }^{11}$ e a redução do consumo alimentar ${ }^{19}$ são contribuidores da perda de peso e da presença de desnutrição.

Esta abordagem de reconhecimento englobando a diversidade dos sinais e sintomas multifatoriais e etiologias subjacentes ao tratamento, como a redução da ingestão alimentar e presença de inflamação, é altamente relevante ao diagnóstico de desnutrição e, consequentemente, para a proposta de tratamento individualizado e eficaz ${ }^{7}$.

Os diferentes \% de perda de peso e a desnutrição são concomitantes com a inflamação gerada pelas patologias presentes no estudo. São doenças altamente catabólicas e possuem resposta elevada para a presença de inflamação sistêmica, consequentemente, levando à piora do estado nutricional ${ }^{20}$.

Conforme observado e associado com esses fatores, o câncer, doenças do trato gastrointestinal, neurológicas e pulmonares apresentaram maior \% de perda de peso. O câncer foi a principal patologia que apresentou maior impacto para a desnutrição, sendo que a chance na análise multivariada de estarem com desnutrição moderada é de 2,21 vezes, e de desnutrição grave, 2,72 vezes. Em outro estudo, usando a AGS-GP, os pacientes com idade $\geq 65$ anos apresentaram alta prevalência (RR 2,16 - 95\% IC $1,87-2,50$ ) da ocorrência de desnutrição grave e qualquer grau de desnutrição comparado com $<50 \operatorname{anos}^{21}$. Kim et al. ${ }^{22}$ e Souza et al. ${ }^{23}$ observaram em pacientes com câncer, quando há aumento de marcadores de inflamação (PCR), maior perda de massa muscular, posteriormente levando a maior perda de peso e risco de desenvolver um quadro de caquexia. Contreras-Bolívar et al. ${ }^{10}$ demonstraram a desnutrição presente em $80,1 \%$ dos pacientes com câncer, estando semelhante aos resultados da ASG quando comparados. Um estudo multicêntrico em pacientes com câncer apontou alta prevalência de desnutrição (avaliado pela PG-SGA) moderada/suspeita $(33,5 \%)$ e grave $(11,8 \%)^{21}$.

A perda de peso não intencional em pacientes hospitalizados associa-se também com alterações psicológicas e fisiológicas provenientes das patologias e/ou tratamentos que resultam aos sintomas de impacto nutricional (SIN), como xerostomia, disgeusia, náuseas e vômitos, entre outros sintomas que limitam ou impedem o paciente de se alimentar. Com esses sintomas, há mudanças na consistência da dieta, levando a alteração do paladar e redução do consumo alimentar, impactando diretamente no estado nutricional ${ }^{24}$.

Neste estudo, o consumo alimentar reduzido/inadequado esteve presente em 83,9\% dos pacientes diagnosticado pelo GLIM com desnutrição moderada e, em $86,9 \%$, com desnutrição grave. Isto é, considerado um valor importante no impacto do estado nutricional, no qual indivíduos com consumo alimentar inadequado apresentam duas vezes mais chances de serem classificados com desnutrição moderada e grave. Estudo realizado com 20 pacientes com diagnóstico de câncer demonstrou que houve redução significativa de todos os grupos alimentares após o início do tratamento ${ }^{25}$. Em outro estudo com 763 pacientes hospitalizados, a redução da ingestão alimentar esteve presente em $53,6 \%$ após o internamento ${ }^{24}$.

Entre as limitações encontradas, destacam-se a não utilização de critérios fenotípicos utilizando técnica de medida da composição corporal, por ser um estudo retrospectivo e no âmbito hospitalar, pois em nossa prática não se utiliza estas avaliações como rotina para determinar o estado nutricional. Desta forma, acreditamos que os dados do histórico do peso, estatura e idade, bem como a patologia podem ser ferramentas de fácil análise da prevalência de desnutrição aplicando a ferramenta GLIM, contribuindo para a validação do novo critério e divulgação dos resultados. 


\section{CONCLUSÃO}

Os pacientes apresentaram grande perda de peso e alta prevalência de desnutrição quando aplicado o GLIM, prevalecendo em indivíduos maiores de 60 anos, principalmente com diagnóstico de câncer e quando relatado o consumo alimentar reduzido/inadequado.

Faz-se necessário novos estudos, de modo a aplicar avaliação nutricional inicial seguido do GLIM, que possam avaliar a perda de peso, perda de apetite, massa magra e comprometimento da capacidade física e, assim, evitar e recuperar os quadros de desnutrição, facilitando a identificação de pacientes em risco nutricional, obtenção de resultados da prevalência, diagnóstico de desnutrição e intervenções.

\section{AGRADECIMENTOS}

Os autores agradecem à Fundação Araucária de Apoio à Pesquisa do Estado do Paraná.

\section{REFERÊNCIAS}

1. Brasil. Ministério da Saúde. Guia alimentar para a população brasileira. Brasília: Ministério da Saúde; 2014.

2. Prieto DB, Leandro-Merhi VA, Mônaco DV, Lazarini ALG. Intervenção nutricional de rotina em pacientes de um hospital privado. Rev Bras Nutr Clin. 2006;21(3):181-7.

3. Cruz LB, Bastos NMRM, Micheli ET. Perfil antropométrico dos pacientes internados em um hospital universitário. Rev HCPA. 2012;32(2):177-81.

4. Rondel ALMA, Langius JAE, de van der Schueren MAE, Kruizenga HM. The new ESPEN diagnostic criteria for malnutrition predict overall survival in hospitalised patients. Clin Nutr. 2018;37(1):163-8.

5. Pedroso CGT, Sousa AA, Salles RK. Cuidado nutricional hospitalar: percepção de nutricionistas para atendimento humanizado. Cien Saude Colet. 2011;16(Supl 1):1155-62.

6. Danone Nutricia. Como prevenir a reinternação após cirurgia [Internet]. [cited 2016 Mar 28]. Available from: http://www. danonenutricao.com.br/alta-hospitalar/noticias/prevenindo-areinternacao-alimentacao-adequada-apos-a-alta-hospitalar

7. Cederholm T, Jensen GL, Correia MITD, Gonzalez MC, Fukushima R, Higashiguchi T, et al. GLIM criteria for the diagnosis of malnutrition: a consensus report from the global clinical nutrition community. Clin Nutr. 2019;38(1):1-9.

8. RaslanM, GonzalezMC,Dias MCG,Paes-BarbosaFC,Cecconello I, Waitzberg DL. Aplicabilidade dos métodos de triagem nutricional no paciente hospitalizado. Rev Nutr. 2008;21(5):553-61.

9. Cederholm T, Jensen GL, Correia MITD, Gonzalez MC, Fukushima R, Higashiguchi T, et al. GLIM criteria for the diagnosis of malnutrition: a consensus report from the global clinical nutrition community. J Clin Nutr. 2019;38(1):1-9.

10. Contreras-Bolívar V, Sánchez-Torralvo FJ, Ruiz-Vico M, González-Almendros I, Barrios M, Padín S, et al. GLIM criteria using hand grip strength adequately predict six-month mortality in cancer inpatients. Nutrients. 2019;11(9). doi: 10.3390/ nu11092043.

11. Cederholm T, Barazzoni R, Austin P, Ballmer P, Biolo G, Bischoff SC, et al. ESPEN guidelines on definitions and terminology of clinical nutrition. Clin Nutr. 2017;36(1):49-64.

12. Muscaritoli M, Lucia S, Farcomeni A, Lorusso V, Saracino $\mathrm{V}$, Barone $\mathrm{C}$, et al. Prevalence of malnutrition in patients at first medical oncology visit: the PreMiO study. Oncotarget. 2017;8(45):79884-96.

13. Fruchtenicht AVG, Poziomyck AK, Reis AMD, Galia CR, Kabke GB, Moreira LF. Inflammatory and nutritional statuses of patients submitted to resection of gastrointestinal tumors. Rev Col Bras Cir. 2018;45(2):e1614.

14. Merhi VAL, Ravelli MN, Ferreira DVM, Oliveira MRM. Relação de concordância entre a avaliação subjetiva global e o índice de massa corporal em pacientes hospitalizados. Alim Nutr. 2007;18(4):375-80.

15. Skeie E, Tangvik RJ, Nymo LS, Harthug S, Lassen K, Viste A. Weight loss and BMI criteria in GLIM's definition of malnutrition is associated with postoperative complications following abdominal resections: results from a National Quality Registry. Clin Nutr [Internet]. 2019. doi: 10.1016/j.clnu.2019.07.003.

16. Powers SK, Lynch GS, Murphy KT, Reid MB, Zijdewind I. Disease-induced skeletal muscle atrophy and fatigue. Med Sci Sport Exerc. 2016;48(11):2307-19.

17. Cruz-Jentoft AJ, Bahat G, Bauer J, Boirie Y, Bruyère O, Cederholm T, et al. Sarcopenia: revised European consensus on definition and diagnosis. Age Ageing. 2019;48(1):16-31.

18. Muscaritoli M, Anker SD, Argilés J, Aversa Z, Bauer JM, Biolo $\mathrm{G}$, et al. Consensus definition of sarcopenia, cachexia and precachexia: joint document elaborated by Special Interest Groups (SIG) "cachexia-anorexia in chronic wasting diseases" and "nutrition in geriatrics". Clin Nutr. 2010;29(2):154-9.

19. Martin L, Kubrak C. How much does reduced food intake contribute to cancer-associated weight loss? Curr Opin Support Palliat Care. 2018;12(4):410-9.

20. Rocha CVS, Freire JAP, Sousa HB, Moura DMS. Perfil nutricional de funcionários de hospital público do município de Picos - Piauí. Rev Interdiscip NovaFapi. 2012;5(1):37-41.

21. Pinho NB, Martucci RB, Rodrigues VD, D’Almeida CA, Thuler LCS, Saunders C, et al. High prevalence of malnutrition and nutrition impact symptoms in older patients with cancer : results of a Brazilian multicenter study. Cancer. 2020;126(1):156-64.

22. Kim EY, Kim YS, Seo JY, Park I, Ahn HK, Jeong YM, et al. The relationship between sarcopenia and systemic inflammatory response for cancer cachexia in small cell lung cancer. PLoS One. 2016;11(8):e0161125. doi: 10.1371/journal.pone.0161125.

23. Souza BJ, Mesquita AU, Meireles AR, Brito JG, Bandeira TE, Rocha JA. Relação entre a atividade inflamatória e o estado nutricional de pacientes com câncer de pulmão. Rev Med UFC. 2019;59(2):9-14.

24. Barbosa AAO, Vicentini AP, Langa FR. Comparação dos critérios da NRS-2002 com o risco nutricional em pacientes hospitalizados. Cien Saude Coletiva. 2019;24(9):3325-34.

25. Véras ID, Santos AF, Ferreira SMS, Oliveira CRR, Costa JG. Alterações orais e ingestão alimentar em pacientes com câncer de cabeça e pescoço em tratamento antineoplásico. Divers J. 2019;4(2):566-79.

Local de realização do estudo: Universidade Estadual do Centro Oeste (UNICENTRO), Guarapuava, PR, Brasil.

Conflito de interesse: Os autores declaram não haver.

Trabalho apresentado no XXIII Congresso Brasileiro de Nutrição Parenteral e Enteral, realizado em Foz do Iguaçu, no período de 20 a 23 de outubro de 2019. 